\title{
Confirmatory Trial
}

National Cancer Institute

\section{Source}

National Cancer Institute. Confirmatory Trial. NCI Thesaurus. Code C142460.

A type of phase 3 clinical trial in which the previously characterized actions of a therapeutic intervention, in an earlier phase trial, are verified. 